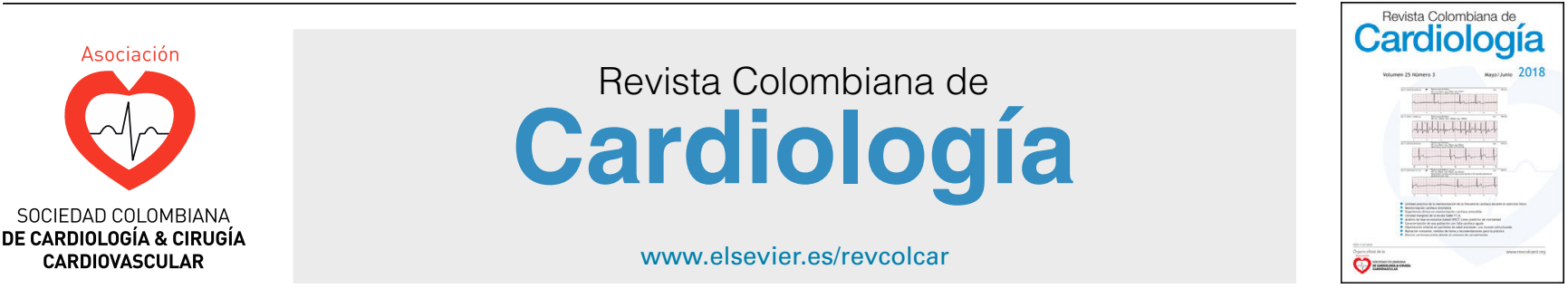

PREVENCIÓN CARDIOVASCULAR

\title{
Evaluación funcional de un programa de entrenamiento físico en pacientes infartados con disfunción sistólica severa del ventrículo izquierdo
}

\author{
Eduardo Rivas Estany ${ }^{a}, *$, Nelson Campos Vera ${ }^{\mathrm{b}}$, José Barrera Sarduy ${ }^{\mathrm{a}}$, \\ Susana Hernández García ${ }^{a}$, Alexander Valdés Martín ${ }^{a}$, Vanessa Peña Bofilla \\ y Elizabeth Prendes Lago ${ }^{a}$
}

\author{
a Instituto de Cardiología y Cirugía Cardiovascular, Centro de Rehabilitación, La Habana, Cuba \\ b Universidad Técnica de Manabí, Manabí, Ecuador
}

Recibido el 13 de abril de 2018; aceptado el 18 de marzo de 2019

Disponible en Internet el 13 de julio de 2019

\author{
PALABRAS CLAVE \\ Entrenamiento físico; \\ Evaluación funcional; \\ Infarto cardíaco; \\ Disfunción sistólica; \\ Rehabilitación \\ cardíaca
}

\begin{abstract}
Resumen
Objetivo: Evaluar la factibilidad y los efectos sobre la capacidad funcional de un programa de entrenamiento físico supervisado, aplicado en pacientes con disfunción sistólica severa del ventrículo izquierdo después de infarto agudo de miocardio.

Métodos: Se estudiaron 37 pacientes, de ambos sexos y sin límites de edad, con diagnóstico de disfunción sistólica severa del ventrículo izquierdo, después de haber sufrido un infarto agudo de miocardio, que consecutivamente se incorporaron al programa ambulatorio del Centro de Rehabilitación del Instituto de Cardiología.

Se hicieron pruebas de esfuerzo máximas limitadas por síntomas con determinación de consumo de oxígeno, ecocardiogramas en reposo y ventriculografías isotópicas en reposo y esfuerzo a los 2, 8 y 18 meses de evolución, y un tiempo medio de seguimiento clínico de 4,1 años. A todos se les prescribió un régimen de entrenamiento físico moderado o intenso, durante un año como mínimo. Se consideró disfunción sistólica severa cuando la fracción de eyección del ventrículo izquierdo fue menor de $35 \%$.

Resultados: Todos los parámetros ergométricos que expresaron capacidad funcional incrementaron significativamente en la evaluación del octavo mes $(p<0,0005)$, permaneciendo invariables a los 18. La fracción de eyección del ventrículo izquierdo media en reposo inicial fue de $28,3 \pm 5,3 \%$, la cual no mostró variaciones significativas con el esfuerzo ni con otros estudios evolutivos. La mortalidad total y la morbilidad de la serie fueron de $10,5 \%$ y $47,3 \%$, respectivamente.

Conclusión: El entrenamiento físico supervisado en pacientes infartados con disfunción sistólica severa de ventrículo izquierdo fue seguro y efectivo, y mejoró su calidad de vida, sin causar efectos negativos sobre la función ventricular.

(C) 2019 Sociedad Colombiana de Cardiología y Cirugía Cardiovascular. Publicado por Elsevier España, S.L.U. Este es un artículo Open Access bajo la licencia CC BY-NC-ND (http:// creativecommons.org/licenses/by-nc-nd/4.0/).
\end{abstract}

\footnotetext{
* Autor para correspondencia.

Correo electrónico: erivas@infomed.sld.cu (E.R. Estany).
} 


\section{KEYWORDS}

Physical training;

Functional

evaluation;

Myocardial infarction;

Systolic dysfunction;

Cardiac rehabilitation

\section{Functional evaluation of a physical training programme in myocardial infarction patients with severe left ventricular systolic dysfunction}

\begin{abstract}
Objective: To evaluate the feasibility and effects on the functional capacity of a supervised physical training programme carried out on patients with severe left ventricular systolic dysfunction after an acute myocardial infarction.

Methods: The study included a total of 37 patients, males and females of any age, with a diagnosis of severe left ventricular systolic dysfunction after having suffered an acute myocardial infarction. They were consecutively included into the ambulatory programme of the Institute of Cardiology Rehabilitation Centre.

Maximum effort tests, limited by symptoms, were performed to determine oxygen consumption. Echocardiograms were also performed at rest, with isotopic ventriculography at rest and then at 2, 8, and 18 months. The mean clinical follow-up was 4.1 years. They were all prescribed to a moderate or intense training programme for at least one year. Severe left ventricular systolic dysfunction was considered when the left ventricular ejection fraction was less than $35 \%$.

Results: All the ergometric parameters that expressed functional capacity increased significantly in the evaluation at 8 months $(P<.0005)$, and remained at 18 months. The initial mean left ventricular ejection fraction at rest was $28.3 \pm 5.3 \%$, which showed no significant changes with effort or in the other evaluation times. The overall mortality and morbidity of the series was $10.5 \%$ and $47.3 \%$, respectively.

Conclusion: Supervised physical training in patients after an acute myocardial infarction and with severe left ventricular systolic dysfunction was safe and effective, and improved the quality of life, without causing negative effects on ventricular function.

(c) 2019 Sociedad Colombiana de Cardiología y Cirugía Cardiovascular. Published by Elsevier España, S.L.U. This is an open access article under the CC BY-NC-ND license (http:// creativecommons.org/licenses/by-nc-nd/4.0/).
\end{abstract}

\section{Introducción}

Hasta hace un poco más de dos décadas, el entrenamiento físico estaba contraindicado en pacientes con insuficiencia cardíaca o con disfunción ventricular izquierda pues en esa época se le atribuía un incremento en el riesgo de muerte o de complicaciones. Paulatinamente, se llevaron a cabo estudios en diversos países, que comenzaron a señalar efectos beneficiosos en estos pacientes, en ausencia de riesgos ${ }^{1,2}$.

Desde principios de la década de los años 90, en el Centro de Rehabilitación Cardiaca del Instituto de Cardiología y Cirugía Cardiovascular de La Habana, se evaluaron los efectos de un programa de entrenamiento físico en pacientes con infarto cardíaco y disfunción de ventrículo izquierdo ligera o moderada, y se obtuvieron resultados satisfactorios ${ }^{3-5}$. En esta ocasión los autores se proponen evaluar el programa actual de entrenamiento físico en pacientes infartados con disfunción sistólica severa de ventrículo después del episodio agudo.

En pacientes con insuficiencia cardíaca o disfunción ventricular izquierda moderada o severa de cualquier etiología, pero en particular en aquellos con cardiopatía isquémica, se requiere el uso de intervenciones combinadas para disminuir la frecuencia de descompensaciones y hospitalizaciones, así como de mortalidad. En adición a tratamientos médicos, intervencionistas o quirúrgicos, el ejercicio físico regular ha demostrado incrementar las condiciones metabólicas y hemodinámicas en pacientes con padecimientos cardíacos, como también en personas asintomáticas portadoras de factores de riesgo.

Para comprender mejor la fisiopatología del entrenamiento físico en pacientes con insuficiencia cardíaca, es preciso resaltar que la principal característica de esta enfermedad es la reducción en la capacidad para realizar ejercicios físicos, como resultado de un flujo insuficiente de sangre hacia los músculos esqueléticos que participan de la actividad, debido no solamente a una reducción del gasto cardíaco ${ }^{1,6,7}$, sino además a un trastorno de la vasodilatación periférica ${ }^{8}$. Los autores hipotetizan que con la práctica de un programa de entrenamiento físico diseñado y supervisado de manera correcta, sería posible revertir algunas de estas alteraciones.

\section{Objetivo}

Evaluar la factibilidad y efectividad de un programa supervisado de entrenamiento físico en pacientes con disfunción sistólica severa del ventrículo izquierdo después de infarto agudo de miocardio.

\section{Material y método}

Se incluyeron en el estudio 37 pacientes con diagnóstico de disfunción sistólica severa del ventrículo izquierdo, después de haber sufrido infarto agudo de miocardio, transmural, de 
cualquier localización, en casos de ambos sexos y sin límite de edad, egresados vivos del Instituto de Cardiología y Cirugía Cardiovascular de La Habana, que consecutivamente se incorporaron al programa ambulatorio del Centro de Rehabilitación Cardíaca de dicha institución ${ }^{9,10}$, el mismo que fue prescrito por lo menos un año. Para el diagnóstico de infarto agudo de miocardio se siguieron los criterios de la Sociedad Europea de Cardiología y del Colegio Americano de Cardiología ${ }^{11}$.

Se indicó a todos los pacientes un régimen de entrenamiento físico de intensidad moderada a elevada $(60-80 \%$ de la frecuencia cardíaca de reserva), con una frecuencia de 3 a 5 sesiones por semana y una duración de 30 a 45 minutos cada una. Todas las sesiones de ejercicios se realizaron bajo la supervisión de Licenciados en Cultura Física y fisioterapeutas, así como bajo control telemétrico de la frecuencia cardíaca y una derivación electrocardiográfica (CM5). El entrenamiento físico consistió en sesiones con intervalos de calistenia, a manera de "calentamiento", de 10 minutos de duración, ejercicios complementarios de acondicionamiento para incrementar fuerza, elasticidad y equilibrio (fig. 1), pedaleo en un cicloergómetro vertical a 50 revoluciones por minuto, caminata o carrera en una correa sinfín (fig. 2), así como empleo de un simulador de remos, todos con la intensidad necesaria para alcanzar y mantener el pulso de entrenamiento predeterminado durante $15 \mathrm{a}$
30 minutos como mínimo. Todos los pacientes se encontraban en clase funcional II-III según la Asociación de Corazón de Nueva York (NYHA su sigla en inglés por New York Heart Association $)^{12}$.

En 22 pacientes $(59,4 \%)$ se hizo trombólisis sistémica con estreptoquinasa recombinante en las primeras 6 horas de evolución del infarto agudo de miocardio. A ninguno se le practicó angioplastia coronaria primaria pero, durante su estadía hospitalaria, a 15 casos $(20,5 \%)$ se les hizo angiografía coronaria con angioplastia e implantación de stent convencional en la arteria culpable del infarto, y a 5 pacientes $(13,5 \%)$ se les implantó un cardiodesfibrilador automático como medida de prevención primaria; igual número de casos recibió el mismo dispositivo durante los primeros 12 meses de evolución con el mismo propósito. Se registró un paciente en choque cardiogénico en las primeras 24 horas de evolución del episodio agudo, quien evolucionó de manera satisfactoria con las medidas farmacológicas instauradas.

El $97,3 \%$ de los casos recibió tratamiento temprano con inhibidores de la enzima convertidora de la angiotensina y el $70,3 \%$ con betabloqueadores. En la tabla 1 se describe otra medicación prescrita durante la hospitalización.

Se hizo seguimiento clínico mediante consultas cardiológicas periódicas en dicho Centro, con un tiempo medio de seguimiento de 4,1 años y un rango de 1 a 7 . Para

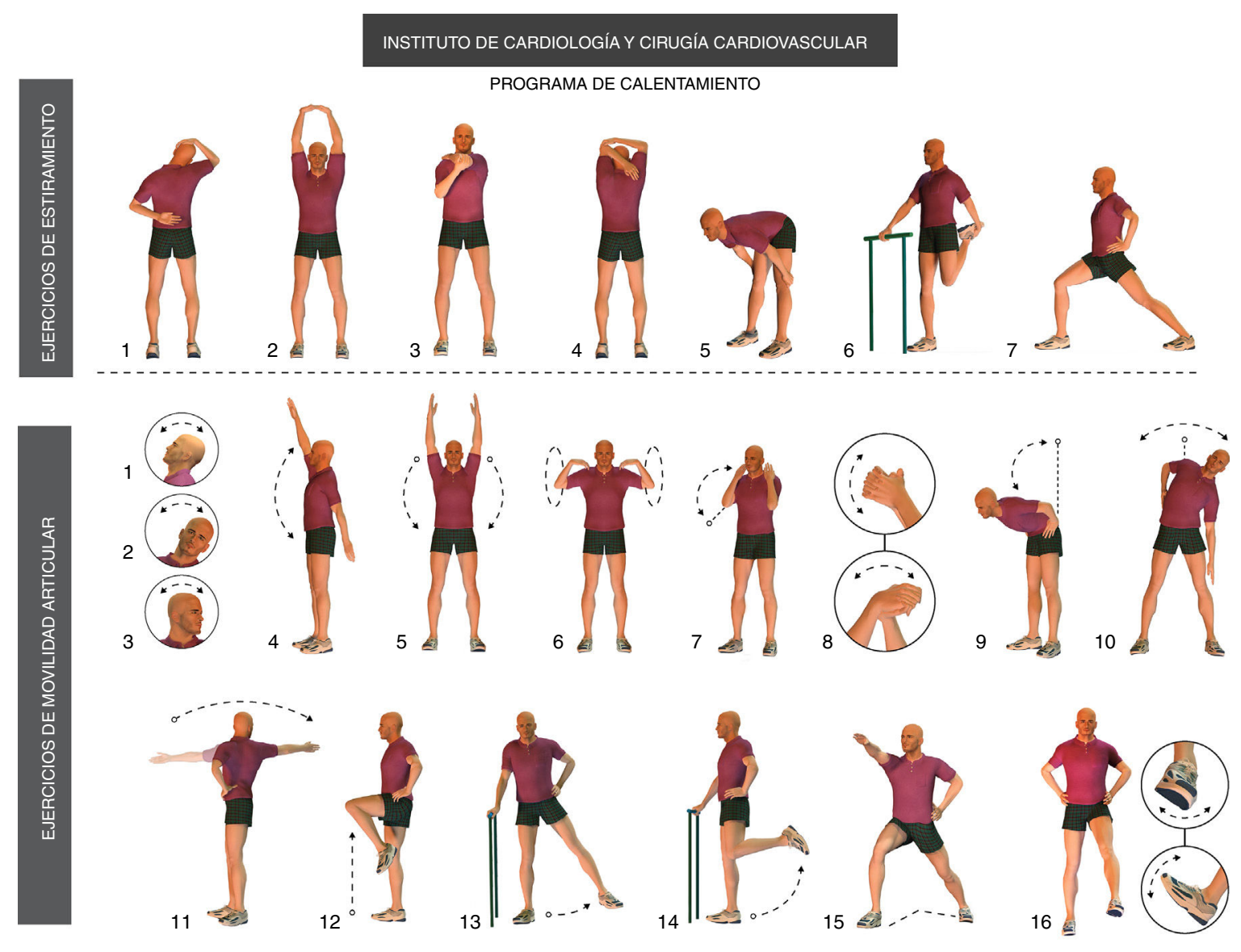

Figura 1 Esquema de ejercicios empleados en los pacientes del estudio durante la fase de calentamiento del programa de entrenamiento físico. Los de la línea superior corresponden a los ejercicios de estiramiento muscular realizados inicialmente, y a continuación los ejercicios de movilidad articular. 


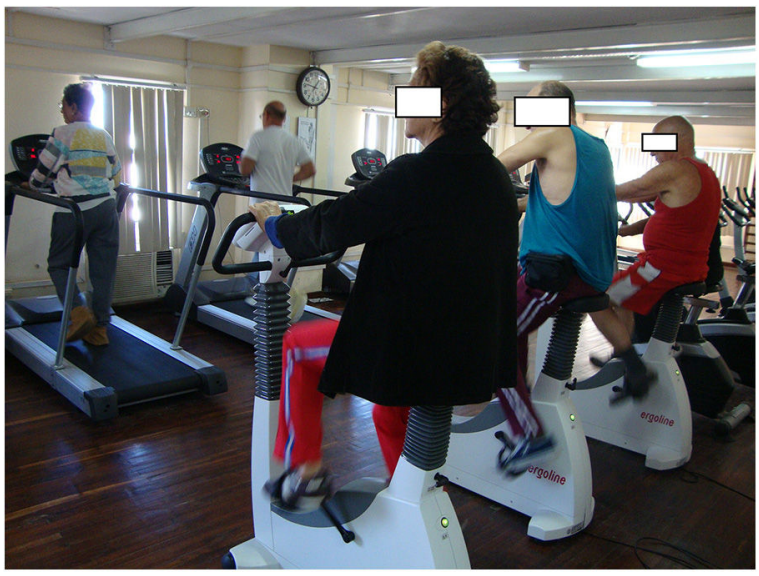

Figura 2 Pacientes infartados, con disfunción ventricular izquierda severa, adscritos a un programa de ejercicios físicos supervisados, en un cicloergómetro vertical de entrenamiento con una velocidad de pedaleo de 50 revoluciones por minuto; otros en una correa sinfín realizando una caminata o carrera, todos con una intensidad de ejercicio necesaria para alcanzar y mantener el pulso de entrenamiento determinado previamente mediante ergometría.

Tabla 1 Características demográficas y clínicas

\begin{tabular}{lll}
\hline $\begin{array}{l}\text { Población total, } \mathrm{n} \\
\text { Sexo femenino, } \mathrm{n}(\%)\end{array}$ & 37 & \\
$\begin{array}{l}\text { Edad media, (años) } \\
\text { infarto agudo de miocardio de }\end{array}$ & 2 & $(5,4)$ \\
$\begin{array}{l}\text { localización anterior, } \mathrm{n}(\%) \\
\text { Medicación: }\end{array}$ & $\mathrm{N}$ & 94,6 \\
Estreptoquinasa recombinante & 22 & $\%$ \\
mediante trombólisis sistémica & & 59,4 \\
IECA & 36 & \\
Betabloqueadores (atenolol) & 26 & 97,3 \\
Nitratos & 21 & 70,3 \\
Diuréticos & 10 & 27,7 \\
Antagonistas del calcio & 4 & 10,8 \\
Antiagregantes plaquetarios & 31 & 83,8 \\
Estatinas & 31 & 83,8 \\
\hline
\end{tabular}

IECA: inhibidores de la enzima convertidora de la angiotensina.

su evaluación funcional se les efectuaron pruebas de esfuerzo máximas limitadas por síntomas, ecocardiogramas bidimensionales, ventriculografías isotópicas en reposo y esfuerzo con Tecnecio 130 a los dos (prueba basal), ocho y dieciocho meses de evolución del infarto agudo de miocardio. Las pruebas de esfuerzo se efectuaron en un cicloergómetro eléctrico vertical marca Siemens-Elema 130 (Alemania); se aplicaron cargas de trabajo continuas, sin pausas de reposo intermedio, a una carga inicial de 25 watts, a manera de calentamiento, e iguales incrementos de 25 watts cada 3 minutos hasta que se alcanzara la frecuencia cardíaca máxima predicha (220 latidos edad) o se presentaran signos o síntomas que constituyeran criterio de interrupción de la prueba. El esfuerzo se realizó bajo monitorización continua de la frecuencia cardíaca y respiratoria y tomas periódicas de la presión arterial, con una frecuencia de pedaleo de 50 revoluciones por minuto; así mismo, se efectuó registro electrocardiográfico de doce derivaciones cada un minuto, que se continuó hasta diez minutos después de finalizado el ejercicio, con el paciente en posición decúbito supino. No se interrumpió el tratamiento médico para la ejecución de las pruebas evaluativas ${ }^{5}$. Se determinó consumo de oxígeno directo mediante el análisis de gases espirados durante el esfuerzo, utilizando un equipo Schiller CS-200 (Suiza) y se aplicó el protocolo usualmente empleado en el Servicio $^{13}$.

En relación con la carga física de trabajo, obtenida mediante ergometría y expresada en watts, se emplearon los siguientes conceptos ${ }^{14,15}$ :

- Carga máxima: mayor carga de trabajo alcanzada

- Capacidad funcional: carga tolerada en relación con la carga predicha, expresada en porcentaje.

- Potencia media: carga media efectuada durante toda la prueba y expresada en watts.

El ecocardiograma bidimensional y en Modo $M$ se llevó a cabo en reposo, en un equipo Toshiba Sonolayer SAC12a (Japón), efectuándose cortes en los ejes longitudinal y transversal en las posiciones paraesternal izquierda, subcostal y supraesternal, con cortes hemiaxiales o de cuatro cámaras en posición apical. Para la determinación de la fracción de eyección de ventrículo izquierdo (FEVI) se empleó el método de Simpson ${ }^{5}$ y se consideró disfunción sistólica severa del ventrículo izquierdo cuando la FEVI en reposo fue menor de $35 \%$.

Se realizaron ventriculografías isotópicas en equilibrio con el fin de precisar la FEVI y la contractilidad regional de paredes en una gammacámara digital Toshiba GCA501 (Japón), en proyección oblicua anterior izquierda y se empleó la metodología comúnmente utilizada en el Centro $^{5,16}$.

Para la recolección de los datos y análisis estadístico se empleó una base de datos SPSS, versión 18.0 para Windows (Chicago, Illinois, Estados Unidos). Se usó, así mismo, una prueba de análisis de varianza para mediciones repetidas multivariadas (ANOVA) a fin de evaluar las diferencias en el tiempo dentro del grupo y la prueba de Chi cuadrado para el estudio de las variables cualitativas o categóricas. Las cifras que se muestran en las tablas 2 y 3 representan los valores medios \pm desviación estándar de la muestra; se consideraron diferencias estadísticamente significativas cuando hubo una $p<0,05$. Se trata de un estudio longitudinal de intervención, prospectivo, abierto, no controlado, en pacientes consecutivos, no seleccionados, los cuales ofrecieron su consentimiento informado antes de ingresar en el estudio, cuyo protocolo había sido previamente evaluado y aprobado por el Comité de Ética de Investigaciones del Consejo Científico de la propia institución.

\section{Resultados}

La edad media de los pacientes fue de $49 \pm 10$ años, con un rango de 30 a 69; de ellos, sólo 2 fueron del sexo femenino $(5,4 \%)$ (tabla 1$) .35$ pacientes tuvieron infarto agudo de miocardio de localización anterior (94,6\%) y 14 historia de un infarto previo $(36,8 \%)$. Los pacientes obtuvieron un $85 \%$ 
de asistencia media a las sesiones de entrenamiento físico, más de $90 \%$ alcanzaron el pulso de entrenamiento durante las sesiones de ejercicios y no se presentaron complicaciones durante estas.

Al evaluar las variables ergométricas que expresan aptitud física, se observó (tabla 2) que todas ellas incrementaron significativamente en la evaluación de los ocho meses en comparación con las del segundo mes, lo que se correspondió con la mejoría significativa del consumo de oxígeno pico $\left(\mathrm{VO}_{2 \text { pico }}\right)$ (de 17,8 a $22,2 \mathrm{ml} / \mathrm{kg} / \mathrm{min}, \mathrm{p}<0,0001$ ) y que ya desde este momento los ubicó en una clase funcional I de acuerdo con la NYHA ${ }^{12}$. La capacidad funcional y la potencia media de trabajo mostraron nuevos incrementos significativos en la prueba de los dieciocho meses de evolución del infarto agudo de miocardio. Así, tanto la carga de trabajo máxima como el doble producto máximo, incrementaron significativamente en la evaluación del octavo mes. El tiempo medio de ejercicio aumentó significativamente de 8 minutos en la prueba basal a 12 y 13 minutos en las pruebas evolutivas $(p<0,01$ y $p<0,05$ respectivamente).

La FEVI media en reposo a los dos meses de evolución, determinada mediante ventriculografía radio-isotópica, fue de $28,3 \%$ y en ejercicio de $29,1 \%$, sin cambios significativos en las evaluaciones posteriores (p: n.s). Los diámetros telediastólicos y telesistólicos del ventrículo izquierdo en reposo, tampoco tuvieron variaciones significativas en cuanto a evolución (tabla 3).

En el tiempo de seguimiento, la serie mostró una mortalidad general de un 10,5\%; el 95\% fue de causa cardiovascular ( 2 casos murieron por tumores malignos). En cuanto a la morbilidad, un 5,3\% tuvo reinfarto cardíaco no fatal y un $10,5 \%$ reingresó por insuficiencia cardíaca, igual porcentaje por angina de pecho y por arritmia ventricular peligrosa, con riesgo inminente para la vida del paciente. Se precisó que el $52,7 \%$ de los casos no presentó complicación alguna durante el tiempo de seguimiento.

\section{Discusión}

Los resultados obtenidos avalan el beneficio de los programas de Rehabilitación Cardíaca multidisciplinar en el pronóstico de pacientes con disfunción de ventrículo izquierdo de origen isquémico, los cuales constituyen un grupo de prevalencia elevada y pronóstico pobre a corto plazo $^{17-19}$. Se trata de uno de los primeros estudios en analizar el impacto de estos programas en este subgrupo de pacientes, así como es uno de los pocos presentes hasta el momento, que ha demostrado el beneficio de un programa de rehabilitación cardiaca multidisciplinario.

En esta investigación, la edad media de los pacientes $(49 \pm 10$ años), estuvo en concordancia con el estudio de Framingham cuyo promedio fue de 50 años. Sin embargo, debe tenerse en cuenta que la población de nuestro estudio la conformaban pacientes con disfunción sistólica severa después del infarto agudo de miocardio. Adicionalmente, de ellos sólo 2 fueron del sexo femenino $(5,4 \%)$ lo cual se correlaciona con los diferentes estudios y las escalas de riesgo en el género masculino como un factor de riesgo independiente para enfermedad coronaria.

En el estudio ELVD en el que se evidenció mejoría de la función ventricular izquierda y atenuación del remodelamiento del miocardio con la práctica de ejercicio físico posterior a un infarto, se documentó mejoría en la capacidad del ejercicio a seis meses después de un programa de entrenamiento físico ${ }^{20}$; en nuestro estudio, al evaluar

Tabla 2 Variables que expresan aptitud física en pruebas de esfuerzo seriadas

\begin{tabular}{|c|c|c|c|c|c|c|c|}
\hline & $\begin{array}{l}\text { Carga } \\
\text { máxima } \\
(\mathrm{w})\end{array}$ & $\begin{array}{l}\text { Potencia } \\
\text { media (w) }\end{array}$ & $\begin{array}{l}\text { Capacidad } \\
\text { funcional (\%) }\end{array}$ & $\begin{array}{l}\text { Doble producto } \\
\text { máximo }(x 100)\end{array}$ & $\begin{array}{l}\text { Doble producto } \\
\text { submáximo } \\
(\times 100)\end{array}$ & $\begin{array}{l}\mathrm{Vo}_{2} \mathrm{pico} \\
(\mathrm{ml} / \mathrm{kg} / \mathrm{mi})\end{array}$ & $\begin{array}{l}\text { IEM } \\
(\times 10)\end{array}$ \\
\hline 2 meses & $80,5 \pm 25$ & $55,7 \pm 5$ & $66,9 \pm 19$ & $22,36 \pm 5,5$ & $20,14 \pm 19,2$ & $17,8 \pm 4,5$ & $11,4 \pm 1,5$ \\
\hline 8 meses & $96,8 \pm 29^{* * * *}$ & $67,1 \pm 6^{* * * *}$ & $77,8 \pm 20^{* * *}$ & $24,34 \pm 6,1^{*}$ & $19,21 \pm 18,1$ & $22,2 \pm 4,3^{* * * *}$ & $12,7 \pm 2,1^{* * * *}$ \\
\hline 18 meses & $100 \pm 28$ & $69,3 \pm 5^{*}$ & $84,6 \pm 16^{* *}$ & $25,29 \pm 7,8$ & $18,58 \pm 18,2$ & $22 \pm 3,5$ & $13 \pm 2,4^{*}$ \\
\hline
\end{tabular}

Tabla 3 Tamaño y función cardíaca evaluados por ecocardiografía y ventriculografía isotópica en reposo y esfuerzo.

\begin{tabular}{lllll}
\hline $\begin{array}{l}\text { Tiempo de } \\
\text { evolución }\end{array}$ & $\begin{array}{l}\text { Diámetro } \\
\text { telediastólico } \\
(\mathrm{mm})\end{array}$ & $\begin{array}{l}\text { Diámetro } \\
\text { telesistólico } \\
(\mathrm{mm})\end{array}$ & $\begin{array}{l}\text { FEVI en reposo } \\
(\%)^{* * *}\end{array}$ & $\begin{array}{l}\text { FEVI en } \\
\text { ejercicio }(\%)^{* * *}\end{array}$ \\
\hline 2 meses & $61,3 \pm 5,6$ & $45,2 \pm 2,8$ & $28,3 \pm 5,3$ & $29,1 \pm 6,6$ \\
8 meses & $60 \pm 4,5$ & $44,5 \pm 2,3$ & $28,5 \pm 6,5$ & $31 \pm 7,1$ \\
18 meses & $59,5 \pm 4,1$ & $44,4 \pm 2,5$ & $27,2 \pm 7,6$ & $30,7 \pm 6,5$ \\
\hline
\end{tabular}

\footnotetext{
* p: n.s.

** Determinado por ecocardiograma. FEVI: fracción de eyección de ventrículo izquierdo determinada mediante ventriculografía isotópica.
} 
las variables ergométricas que expresan aptitud física, se observó que todas ellas incrementaron significativamente en la evaluación de los 8 meses en comparación con la del segundo mes, lo que se correlacionó con la mejoría significativa del $\mathrm{VO}_{2 \text { pico }}$ (de 17,8 a 22,2 $\mathrm{ml} / \mathrm{kg} / \mathrm{min}, \mathrm{p}<0,0001$ ) y que ya desde este momento los ubicó en una clase funcional I de acuerdo con la NYHA ${ }^{12}$. La determinación del $\mathrm{VO}_{2 \text { pico }}$ mediante el análisis de gases espirados durante el esfuerzo físico, representa una de las principales variables que expresan la capacidad funcional del sujeto evaluado ya que refleja la función del ventrículo izquierdo y por ende, posee un valor notable en la estratificación de riesgo y en el pronóstico de pacientes, más aun en aquellos con disfunción de ventrículo izquierdo o insuficiencia cardíaca ${ }^{21,22}$. Es, además, un parámetro evaluativo esencial que expresa la efectividad del régimen de entrenamiento físico empleado como parte de un programa de rehabilitación cardiaca ${ }^{23}$, de ahí que se pueda considerar que el protocolo de ejercicios evaluado en esta investigación arrojó resultados favorables en el grupo de pacientes estudiados.

La capacidad funcional y la potencia media de trabajo mostraron nuevos incrementos significativos en la prueba de los dieciocho meses de evolución del infarto agudo de miocardio. Así, tanto la carga de trabajo máxima como el doble producto máximo, aumentaron de manera significativa en la evaluación del octavo mes. Por su parte, el tiempo medio de ejercicio aumentó significativamente de 8 minutos en la prueba basal a 12 y 13 minutos en las pruebas evolutivas $(p<0,01$ y $p<0,05$ respectivamente), lo cual se correlaciona con el impacto reportado en diferentes estudios como el EXERT para prevenir la incapacidad resultante de la enfermedad coronaria, principalmente en población vulnerable como ancianos y aquellos que involucran ocupaciones que requieren algún esfuerzo físico ${ }^{24}$. Haykowsky et al. ${ }^{25}$ reportaron en un conocido metaanálisis, efectos beneficiosos sobre el remodelamiento ventricular izquierdo después de infarto agudo de miocardio cuando el entrenamiento físico se inició tan temprano como desde una semana después del episodio agudo y tuvo una duración mayor de tres meses; estos resultados coinciden con otro estudio de nuestro grupo ${ }^{14}$, publicado en 2013, en una serie de pacientes rehabilitados con ejercicio físico moderado o intenso y remodelamiento de ventrículo izquierdo después de un infarto agudo de miocardio de pared anterior en quienes el $41,1 \%$ sufría disfunción sistólica severa del ventrículo izquierdo, con FEVI menor del $40 \%$.

En el tiempo de seguimiento, la serie mostró una mortalidad general de un 10,5\%, donde el $95 \%$ fue de causa cardiovascular (2 casos murieron por tumores malignos). En cuanto a la morbilidad, un 5,3\% tuvo reinfarto cardíaco no fatal y un 10,5\% reingresó por insuficiencia cardíaca, igual porcentaje por angina de pecho y por arritmia ventricular peligrosa, con riesgo inminente para la vida del paciente. A todos ellos se les aplicaron las normas y protocolos de actuación establecidas en la Institución y ninguno falleció durante la nueva hospitalización. Se precisó que el 52,7\% de los casos no presentó complicación alguna durante el tiempo de seguimiento, lo cual concuerda con la evidencia sólida del beneficio de los programas de rehabilitación cardiovascular en pacientes con disfunción del ventrículo izquierdo e insuficiencia cardíaca en mejoría de la calidad de vida ${ }^{26-28}$ y reducción de ingresos hospitalarios ${ }^{29}$, sin aparente beneficio sobre la mortalidad ${ }^{2}$. No obstante, metaanálisis más recientes señalan efectos favorables sobre la mortalidad en pacientes isquémicos rehabilitados, incluyendo aquellos con disfunción ventricular izquierda e insuficiencia cardíaca, independientemente del tipo de programa de entrenamiento físico realizado ${ }^{30,31}$. En los últimos años se está considerando además la indicación de entrenamiento físico a intervalos de alta intensidad, que ha ofrecido resultados satisfactorios en cuanto a una más rápida mejoría de la capacidad funcional y por ende de la calidad de vida del paciente con insuficiencia cardíaca, en ausencia de complicaciones ${ }^{32}$.

Finalmente, de acuerdo con los resultados del trabajo que aquí se publica, es posible concluir que el programa de entrenamiento físico evaluado en pacientes con disfunción sistólica severa del ventrículo izquierdo después de infarto agudo de miocardio, proporcionó mejoría de los parámetros ergométricos que determinaron aptitud física, sin haberse precisado cambios de la FEVI en reposo o esfuerzo; por tanto, el incremento de la capacidad física se atribuye a adaptaciones periféricas y músculo-esqueléticas ocasionadas por el ejercicio físico ${ }^{7}$.

\section{Limitaciones}

La principal consistió en no contar con un grupo control para realizar una comparación intergrupal. En la actualidad, cumpliendo las guías de actuación de la Institución en pacientes con infarto agudo de miocardio, existen limitaciones éticas para excluir a estos casos de los programas de rehabilitación cardiaca; por tanto, se decidió realizar una evaluación intra-grupo. Otra de las limitaciones se refiere al sexo, pues sólo dos mujeres integraron la muestra.

\section{Conclusiones}

El programa de entrenamiento físico supervisado en pacientes con infarto agudo de miocardio complicados con disfunción sistólica severa del ventrículo izquierdo, fue seguro y efectivo, ya que mejoró su calidad de vida, sin generar efectos negativos sobre la función ventricular o la morbi-mortalidad en estos casos portadores de un pronóstico desfavorable.

\section{Conflicto de intereses}

Ninguno.

\section{Bibliografía}

1. Piña IL, Apstein CS, Balady GJ, Belardinelli R, Chaitman BR, Duscha $\mathrm{BD}$, et al. Exercise and heart failure. a statement from the American Heart Association Committee on Exercise. Rehabilitation and Prevention. Circulation. 2003;107:1210-25.

2. O'Connor CM, Whellan DJ, Lee KL, Keteyian SJ, Cooper LS, Ellis SJ, et al. Efficacy and safety of exercise training in patients with chronic heart failure. HF- ACTION randomized controlled trial. JAMA. 2009;301:1439-50.

3. Barrera Sarduy JD, Rivas Estany E, Álvarez Gómez JA, Echarte Martínez JC. Rehabilitación cardíaca en la disfunción del 
ventrículo izquierdo. Rev Cubana de Cardiología y Cirugía Cardiovascular. 1996;10:32-6.

4. Rivas Estany E, Castillo Alfonso M, Sin Chesa C, Peix González A, Hernández González R. Efectos del entrenamiento físico en pacientes con disfunción sistólica de ventrículo izquierdo después del infarto miocárdico. Rev Latina Cardiol. 1996;17: $1-5$.

5. Rivas Estany E, Castillo Alfonso M, Rodríguez Nande L, Ponce Vicente F, Peix González A, Abreu Cruz JE, et al. Empleo de pruebas no invasivas en la caracterización de pacientes con disfunción de ventrículo izquierdo después del infarto miocárdico. Su valor en la Rehabilitación Cardíaca. Cardiología Intercontinental. 1998;7:32-6.

6. Sullivan MJ, Hawthorne MH. Exercise intolerance in patients with chronic heart failure. Prog Cardiovasc Dis. 1995;38:1-22.

7. Rivas Estany E, Hernández García S. entrenamiento físico en la insuficiencia cardíaca crónica: fisiopatología y evolución clínica. Medwave. 2016;16 Suppl 4:e6517, http://dx.doi.org/10.5867/medwave.2016.6517.

8. Braunwald E. Heart failure. J Am Coll Cardiol HF. 2013;1:1-20.

9. Rivas Estany E, Barrera Sarduy J, Sixto Fernández S, Rodríguez Nande LM. Kesser García. Programa Cubano de Rehabilitación Cardíaca. Resultados. Rehabilitación (Madr.). 2013;47:238-44.

10. Rivas Estany E. Rehabilitación cardíaca prolongada. En: Maroto Montero JM, de Pablo Zarzosa C, editores. Rehabilitación cardiovascular, Capítulo 32. Madrid: Editorial Panamericana; 2011. p. 463-72.

11. Alpert JS, Thygesen K, Antman E, Bassand JP. Myocardial infarction redefined-a consensus document of The Joint European Society of Cardiology/American College of Cardiology Committee for the redefinition of myocardial infarction. J Am Coll Cardiol. 2000;36:959-69.

12. Jessup M, Abraham WT, Casey DE, Feldman AM, Francis GS, Ganiats TG, Marvin A, et al., 2009 Focused Update: ACCF/AHA Guidelines for the Diagnosis and Management of Heart Failure in Adults: A Report of the American College of Cardiology Foundation/American Heart Association Task Force on Practice Guidelines Developed in Collaboration With the International Society for Heart and Lung Transplantation. J Am Coll Cardiol. 2009;53:1343-82.

13. Gómez López N, Rivas Estany E, Crespo Fernández FF. Análisis de gases espirados durante la prueba de esfuerzo: caracterización de resultados en sujetos sin patología cardiovascular. Rev Cubana Cardiol Cir Cardiovasc. 2014;20:166-75.

14. Rivas-Estany E, Sixto-Fernández S, Barrera-Sarduy JD, Hernández-García S, González Guerra R, Stusser-Beltranena R. Efectos del entrenamiento físico de larga duración sobre la función y remodelación del ventrículo izquierdo en pacientes con infarto de miocardio de pared anterior. Arch Cardiol Mex. 2013;83:167-73.

15. Fletcher GF, Ades PA, Kligfield P, Arena R, Balady GJ, Bittner VA, et al. Exercise standards for testing and training: a scientific statement from the American Heart Association. Circulation. 2013;128:873-934, http://dx.doi.org/10. $1161 /$ CIR.0b013e31829b5b44.

16. Peix A, Karthikeyan G, Massardo T, Kalaivani M, Patel CM, Pabon $\mathrm{L}$, et al. Value of intraventricular dyssynchrony assessment by gated-SPECT myocardial perfusion imaging in the management of heart failure patients undergoing cardiac resynchronization therapy (VISION-CRT). J Nuclear Cardiol. 2019, http://dx.doi.org/10.1007/s12350-018-01589-5.

17. Meta-analysis Global Group in Chronic Heart Failure (MAGGIC). The survival of patients with heart failure with preserved or reduced left ventricular ejection fraction: an individual patient data meta-analysis. Eur Heart J. 2012;33:1750-7.

18. Stewart S, Maclntyre K, Hole DJ, Capewell S, McMurray JJ. More malignant than cancer? Five-year survival following a first admission for heart failure. Eur J Heart Fail. 2001;3:315-22.

19. Stewart S, Ekman I, Ekman T, Odén A, Rosengren A. Population impact of heart failure and the most common forms of cancer: a study of 1162309 hospital cases in Sweden (1988 to 2004). Circ Cardiovasc Qual Outcomes. 2010;3:573-80.

20. Ades PA. Cardiac rehabilitation and secondary prevention of coronary heart disease. N Engl J Med. 2001;345:892-902.

21. Corrà U, Agostoni PG, Anker SD, Coats AJS, Crespo Leiro MG, de Boer RA, et al. Role of cardiopulmonary exercise testing in clinical stratification in heart failure. A position paper from the Committee on exercise physiology and training of the Heart Failure Association of the European Society of Cardiology. Eur J Heart Fail. 2018;20:3-15, http://dx.doi.org/10.1002/ejhf.979.

22. Rivas Estany E, Gómez López N. Objective assessment of functional capacity: the role of cardiopulmonary exercise testing. CorSalud. 2013;5:232-6.

23. Valdés Martín A, Rivas Estany E, Antuña Aguilar T, Echevarría Sifontes LA. Utilidad de la Ergoespirometría en el diagnóstico y evaluación de las enfermedades cardiovasculares. Rev Cubana Cardiol Cir Cardiovasc. 2016;22:47-53.

24. McKelvie R, Teo K, Roberts R, McCartney N, Humen D, Montague $T$, et al. Effect of exercise training in patient with heart failure: The Exercise Rehabilitation Trial (EXERT). Am Heart J. 2002;144:23-30.

25. Haykowsky M, Scott J, Esch B, Schopflocher D, Myers $\mathrm{J}$, Paterson I, et al. A meta-analysis of the effects of exercise training on left ventricular remodeling following myocardial infarction: start early and go longer for greatest exercise benefits on remodeling. Trials. 2011;12:92, http://dx.doi.org/10.1186/1745-6215-12-92.

26. Doimo S, Enrico E, Piepoli M, Barbati G, Antonini-Canterin F, Bernardi G, et al. Impact of ambulatory cardiac rehabilitation on cardiovascular outcomes: a long-term follow-up study. Eur Heart J. 2019;40:678-85, http://dx.doi.org/10. 1093/eurheartj/ehy417.

27. Parsa A, Sadeghi M, Roghani F, Yazdekhasti S, Golshani J, Khani A. Effects of changes in myocardial dysfunction on quality of life in patients undergoing coronary angioplasty after cardiac rehabilitation. Iranian Heart Journal. 2018;19:52-60.

28. de Gregorio C. Physical Training and Cardiac Rehabilitation in Heart Failure Patients. Adv Exp Med Biol. 2018;1067:161-81, http://dx.doi.org/10.1007/5584_2018_144.

29. Gheorghiade M, Vaduganathan M, Fonarow GC, Bonow RO. Rehospitalization for Heart Failure: Problems and Perspectives. J Am Coll Cardiol. 2013;61:391-403.

30. Palmer K, Bowles K-A, Paton M, Jepson M, Lane R. Chronic heart failure and exercise rehabilitation: a systematic review and meta-analysis. Arch Phys Med Rehabil. 2018;99: 2570-82.

31. Santiago de Araújo Pio C, Marzolini S, Kin R, Pakosh M, Grace SL. Effect of cardiac rehabilitation dose on mortality and morbidity: A systematic review and meta-regression analysis. Mayo Clin Proc. 2017;92:1644-59.

32. Giallauria F, Piccioli L, Vitale G, Filippo M, Sarullo FM. Exercise training in patients with Chronic Heart Failure: a new challenge for Cardiac Rehabilitation Community. Monaldi Archives for Chest Disease. 2018;;88:987, http://dx.doi.org/10.4081/monaldi.2018.987. Archives for Chest Disease 2018; volume 88:987 fo:9. 